\title{
The correlates and dynamics of COVID-19 vaccine-specific hesitancy ${ }^{1}$
}

\author{
Eric Merkley ${ }^{a *}$, and Peter John Loewen ${ }^{\mathrm{a}, \mathrm{b}}$ \\ ${ }^{a}$ Department of Political Science, University of Toronto, Toronto, Canada \\ ${ }^{b}$ Munk School of Global Affairs and Public Policy, University of Toronto, Toronto, Canada \\ * Corresponding author: Eric Merkley (eric.merkley@utoronto.ca) \\ Accepted for publication at V accine
}

\begin{abstract}
Most work on COVID-19 vaccine hesitancy has focused on its attitudinal and demographic correlates of individuals, but the characteristics of vaccines themselves also appear to be important. People are more willing to take vaccines with higher reported levels of efficacy and safety. Has this dynamic sparked comparative hesitancy towards specific COVID-19 vaccines? We conduct a series of cross-sectional survey experiments to test for brand-based differences in perceived effectiveness, perceived safety, and vaccination intention. Examining more than 6,200 individuals in a series of cross-sectional surveys, we find considerably more reluctance to take the AstraZeneca and Johnson \& Johnson vaccines compared to those from Pfizer and Moderna if offered, despite all vaccines being approved and deemed safe and effective by a federal regulator. Comparative hesitancy towards these vaccines grew over the course of our fielding as controversy arose over their link to extremely rare, but serious side effects. Comparative vaccine-specific hesitancy is strongest among people who are usually most open to mass vaccination efforts. Its effects are substantial: most respondents reported a willingness to wait months for their preferred vaccine rather than receive either the AstraZeneca or Johnson \& Johnson vaccine immediately. Our findings call for additional research on the determinants and consequences of COVID-19 vaccine-specific hesitancy and communication strategies to minimize this challenge.
\end{abstract}

\footnotetext{
${ }^{1}$ We thank members of the Policy, Elections and Representation lab at the Munk School of Public Policy and Global Affairs for their helpful feedback on this project. We also thank the University of Toronto, 19toZero and the Department of Canadian Heritage for funding the surveys in which these experiments were included.
} 


\section{Introduction}

A globally-scaled mass vaccination campaign is integral to containing the COVID-19 pandemic. As new variants emerge and as protection from initial vaccines wane, it will also be necessary to distribute follow-up "booster" shots. Encouraging continued uptake of COVID-19 vaccines by members of the public is a vital and urgent public health issue.

A growing body of research has been dedicated to understanding the sources and dynamics of COVID-19 vaccine hesitancy, and how it complicates efforts at mass vaccination. Most of this research, however, aims to understand hesitancy towards COVID-19 vaccines in general. It is also likely, however, that people have preferences over currently available vaccine options and exhibit considerable hesitancy towards specific vaccines that are influenced by objective features of competing vaccines or communications surrounding them. Depending on the availability of various vaccines in different national contexts, this could be deeply problematic for mass vaccination efforts in its own right.

This paper uses Canada as a case study of these dynamics. As of January 2022, the national regulator, Health Canada, has approved four COVID-19 vaccines for distribution: Pfizer, Moderna, AstraZeneca, and Johnson \& Johnson, each with different recommended usage guidelines, levels of availability, and safety and efficacy profiles. We ask three principal research questions: 1) to what degree do people exhibit hesitancy towards specific vaccines? 2) How does this hesitancy vary over time in response to the communication environment? And 3) which groups of citizens are more likely to exhibit this hesitancy?

We answer these questions with a study included in repeated cross-sectional surveys of adult Canadian citizens, fielded between February and May 2021 as Canada's mass vaccination campaign began a large-scale roll-out. Importantly, the initially limited supply of available vaccines meant that individuals were sometimes unable to choose a particular brand, though brand choice eventually became possible, depending on an individual's province of residence and other individual-level factors. The study features an experiment asking respondents to rate the effectiveness and safety of a randomly assigned vaccine, and indicate their willingness to take the vaccine if offered, conducted over three waves as controversy emerged over the AstraZeneca and Johnson \& Johnson vaccines. We rely on comparisons between individuals to infer vaccine-specific hesitancy.

We find substantial vaccine-specific hesitancy in the Canadian context. Respondents reported comparatively more hesitancy towards the AstraZeneca and Johnson \& Johnson vaccines, and this hesitancy grew over the course of fielding as controversy related to these vaccines arose. Second, we show that people's beliefs about the safety of vaccines became an increasingly important influence on people's willingness to take them if offered, especially among respondents who were randomly assigned AstraZeneca or Johnson \& Johnson. We also show that comparative aversion to the AstraZeneca and Johnson \& Johnson vaccines is stronger for those who are typically most supportive of vaccines and is intense: a sizable segment of the population is willing to wait months to receive their preferred vaccine over those from AstraZeneca or Johnson \& Johnson. 


\section{COVID-19 vaccine hesitancy}

Ensuring continued widespread uptake of vaccines is vitally important to controlling the COVID-19 pandemic. However, we know from history that vaccination efforts are often met with resistance in some quarters of society, despite being one of the most important public health advances in history (Wolfe \& Sharpe, 2002). A large body of scholarly work has sought to identify the factors that make people more vaccine hesitant - a reluctance or refusal of an individual to receive a vaccine for themselves or their children - or prone to endorse vaccine misinformation and antivaccine policy attitudes.

Several important lessons have emerged from this line of research. Ideological conservatives appear to be more vaccine hesitant in the United States than liberals (Baumgaertner, Carlisle \& Justwan, 2018), which may not have been true in the past. Republican supporters are also less likely to hold accurate beliefs about vaccines, with possible downstream consequences on vaccine policy preferences (Joslyn \& Sylvester, 2019; Motta, 2021b). Support for specific vaccines can also decrease in response to politicization of a given vaccine in the news media (Fowler \& Gollust, 2015). Lower knowledge levels are also associated with greater confidence that one knows more than vaccine experts, which is in turn associated with anti-vaccine policy attitudes - a Dunning-Kruger effect (Motta, Callaghan \& Sylvester, 2018). Distrust in scientific authorities is also heavily connected to vaccine misinformation endorsement, as is social media use (Stecula, Kuru \& Jamieson, 2020), consistent with a wide range of work documenting vaccine misinformation online (Elkin, Pullon \& Stubbe, 2020; Jamison et al., 2020; Keelan et al., 2007; Shah et al., 2019; Tang et al., 2021).

A growing body of work examining the correlates of COVID-19 vaccine hesitancy has arrived at very similar conclusions. Hesitancy is found among ideological conservatives, at least in the American context, as well as those with low trust in experts. It is also higher among racialized minorities and women, those with more hesitancy towards childhood vaccines, and those who are skeptical of the seriousness of the COVID-19 pandemic (Callaghan et al., 2021; Motta, 2020). These findings also line up with research examining the correlates of compliance with other public health guidelines like mask wearing and social distancing (Allcott et al., 2020; Gadarian, Goodman \& Pepinsky, 2021; Merkley \& Loewen, 2021; Pennycook et al., 2021).

The case of COVID-19 vaccines, however, is unique in one other way: there are multiple vaccines on offer each with different characteristics and safety and efficacy profiles. The availability of these vaccines will vary over time and across national contexts. The above work examines attitudinal and demographic correlates of hesitancy towards COVID-19 vaccines as an aggregate category, but the characteristics of vaccines may also matter for hesitancy towards specific vaccines.

There are good reasons to expect people may have hesitancy towards specific COVID-19 vaccines. Trial data for COVID-19 vaccines by AstraZeneca and Johnston \& Johnston indicated they were less effective at preventing symptomatic infection than those by Pfizer and Moderna, though they were reported to all be similarly effective at preventing severe disease and death (Advisory Committee on Immunization Practices, 2021; Teo, 2021). More recently, public health agencies in some countries raised alarms about a link between the AstraZeneca and Johnson \& 
Johnson vaccines and rare, but severe adverse events, which was widely reported in the news media. These dynamics may have induced vaccine-specific hesitancy. In short, people may have formed preferences over the range of available COVID-19 vaccines and developed comparative hesitancy towards some of them based on discourse surrounding the safety and efficacy characteristics of these vaccines.

There has been some work exploring how the characteristics of bypothetical COVID-19 vaccines affect hesitancy. People are less willing to take foreign-manufactured vaccines, as well as those with weaker safety and efficacy profiles (Kaplan \& Milstein, 2021; Kreps et al., 2020; Motta, 2021a). But these studies were done before it was clear which COVID-19 vaccines were going to be on offer. Merkley \& Loewen (2021) focus on identifying communication strategies to improve willingness to take the AstraZeneca and Johnson \& Johnson vaccines, but do not identify how hesitancy towards these vaccines compares to those from Pfizer or Moderna, how that has changed over time, and who exactly is most likely to differentiate by brand. This is our focus in this paper.

\section{Materials and Methods}

Our research was approved by the University of Toronto Social Sciences, Humanities and Education Research Ethics Board (protocol no. 38251). All respondents included in the following analyses provided their informed consent. Our research was fielded in three cross-sectional waves February 23-March 1, 2021 (N=2,495), March 17-23, 2021 (N=2,511) and April 15-20, 2021 $(\mathrm{N}=1,455)$ - conducted on non-probability samples of adult Canadian citizens from the online panel provider Dynata. Quotas in each wave were set on age (i.e., 18/34, 35/54, and 55+), gender (i.e., male, female), region (i.e., Atlantic, Quebec, Ontario, and West), and language (i.e., English, French) to match population benchmarks in the 2016 Canadian census. Table S1 of the Supplementary Materials provides a breakdown in the demographics of each of the three surveys along with the population benchmark. ${ }^{2}$

We conducted a four condition between-subjects experiment. Respondents were randomly assigned into four groups: Pfizer, Moderna, AstraZeneca, and Johnson \& Johnson. ${ }^{3}$ They received the following three questions where the brand of the vaccine was piped into the text based on their assigned condition: 1) If you were offered the [insert brand] coronavirus vaccine, how likely would you be to take it? (very likely, somewhat likely, not very likely, not at all likely); 2) How would you rate the effectiveness of the [insert brand] coronavirus vaccine? (very effective, somewhat effective, not very effective, not at all effective); and 3) How would you rate the safety of the [insert brand] coronavirus vaccine? (very safe, somewhat safe, not very safe, not at all safe). All of our outcomes are rescaled from 0-1 where 1 indicates the most positive vaccine evaluations.

\footnotetext{
2 The mass vaccination campaign in Canada began to roll out after our second wave. Consequently, we began tracking the number of people who reported their first COVID-19 vaccine dose as of wave 3. In one departure from preregistration, we remove 213 respondents from our third wave who self-reported having been vaccinated. The results from the following analyses, however, remain virtually identical when including these respondents.

${ }^{3}$ In the first wave Johnson \& Johnson was excluded because it was not yet approved by Health Canada.
} 
We pre-registered expectations that intention (H1), perceived effectiveness (H2), and perceived safety (H3) should be lower for respondents in the AstraZeneca and Johnson \& Johnson treatment conditions compared to those in the Moderna and Pfizer conditions. Information on the comparatively lower efficacy of the AstraZeneca and Johnson \& Johnson vaccines likely weakened confidence in their effectiveness, while media coverage of a link between these vaccines and serious side effects reduced perceptions of their safety. These changing perceptions, in turn, reduced willingness to receive these vaccines if offered. ${ }^{5}$

We test our hypotheses with an independent samples t-test $(1=$ AstraZeneca/Johnson \& Johnson, $0=$ Pfizer/Moderna). We also present the model predictions for the following equation with survey wave fixed effects $(\mathrm{X})$ estimated using Ordinary Least Squares regression to illustrate the differences across our three outcomes for each vaccine:

outcome $=\alpha+\beta_{1}$ moderna $+\beta_{2}$ aztrazeneca $+\beta_{3} j \& j+X+\varepsilon$

All significance tests are two-tailed. We use a p-value threshold of 0.05 to determine statistical significance with HC2-robust standard errors. All analyses are conducted using STATA version 16.

Events transpired during our fielding that likely influenced vaccine intention and perceptions of their safety and efficacy above and beyond baseline expectations derived from initial safety and efficacy trials. Wave 1 (February 23-March 1) was conducted before safety concerns arose with the AstraZeneca vaccine. Health Canada approved AstraZeneca for adults 18 and older on February 26 in the middle of fielding. Wave 2 (March 17-23) was conducted after considerable controversy arose with the AstraZeneca vaccine - specifically its link to rare, but serious blood clotting episodes. Denmark suspended administration of the AstraZeneca vaccine on March 11 and Germany and France followed suit on March 14. All told, a dozen European countries suspended AstraZeneca vaccine administration in between waves 1 and 2, while Health Canada maintained their safety and effectiveness. Most of the skepticism towards the AstraZeneca vaccine expressed by public health authorities, to this point, was from public health agencies in other countries.

During the interval between waves 2 (March 17-23) and 3 (April 15-20), growing skepticism of the AstraZeneca vaccine was expressed by domestic health authorities. On March 29 the National Advisory Committee on Immunization (NACI) recommended suspending AstraZeneca administration for those under the age of 55 due to blood clotting concerns, and the provinces

\footnotetext{
${ }^{4}$ The pre-registration for our studies can be found here: (https://osf.io/74e2x). The first wave was conducted as a pilot study prior to registration.

${ }^{5}$ We cannot directly shed light on the causal mechanism linking COVID-19 vaccine brand to intention with these design (see Bullock et al. (2010) for challenges of causal inference with mediation analysis). That being said, we include a path analysis in the Supplementary Materials that descriptively shows the direct and indirect effects of our COVID-19 vaccine brand treatment on intention to vaccinate. These analysis show that $75 \%$ of the effect of the brand treatment on intention runs indirectly through changes in perceived effectiveness and safety of the vaccine. More details on this analysis and its limitations can be found in the Supplementary Materials.
} 
followed this recommendation immediately. ${ }^{6}$ Health Canada, however, continued to maintain that the benefits of the vaccine outweighed the risks - an announcement they made on April 14. In addition, on April 13 the U.S. Food and Drug Administration also recommended a pause in the administration of the Johnson \& Johnson vaccine due to similar safety concerns. Our period of fielding allow us to observe potential dynamics in vaccine-specific hesitancy over this period.

We estimate a series of models where we regress our outcome variables on the brand conditions, the survey waves, and interactions between the brand conditions and survey waves as follows:

outcome $=\alpha+\beta_{1}$ moderna $+\beta_{2}$ aztrazeneca $+\beta_{3} j \& j+\beta_{4}$ wave $2+\beta_{5}$ wave $3+$ $\beta_{6}$ moderna $*$ wave $2+\beta_{7}$ moderna $*$ wave $3+\beta_{8}$ astrazeneca $*$ wave $2+$ $\beta_{9}$ astrazeneca $*$ wave $3+\beta_{10} j$ \& $*$ wave $3+\varepsilon$

We display the marginal effects of each brand (reference $=$ Pfizer) across each wave of our study to illustrate dynamics over the course of our fielding.

We expect reductions in all three of our outcomes for the AstraZeneca and Johnson \& Johnson vaccines as a result of communication surrounding these vaccines. But, this attention was primarily focused on rare side effects related to the AstraZeneca and Johnson \& Johnson vaccines, rather than efficacy concerns. In so doing, the communication environment may have primed safety perceptions. We may observe a growing association between safety perceptions and intention over the course of fielding. We estimate the following equation:

intention $=\alpha+\beta_{1}$ effectiveness $+\beta_{2}$ safety $+\beta_{3}$ wave $2+\beta_{4}$ wave $3+$ $\beta_{5}$ effectiveness $*$ wave $2+\beta_{6}$ effectiveness $*$ wave $3+\beta_{7}$ safety $*$ wave $2+\beta_{8}$ safety $*$ wave $3+\varepsilon$

Increased communication around vaccine safety may have primed safety considerations across the board, or it may have increased the importance of these considerations only for the vaccines at the centre of the emerging controversy. So, we estimate the following equation which allows us to evaluate this priming effect across treatment conditions (i.e., Pfizer/Moderna vs. AstraZeneca/Johnson \& Johnson):

\footnotetext{
${ }^{6}$ Importantly, NACI operates as an advisory board and not a regulator. It existed before the COVID-19 pandemic, and previously focussed principally on childhood vaccinations.
} 
intention

$=\alpha+\beta_{1}$ effectiveness $+\beta_{2}$ safety $+\beta_{3}$ wave $2+\beta_{4}$ wave $3+\beta_{5}$ astrazeneca $/ j \& j$

$+\beta_{6}$ effectiveness $*$ wave $2+\beta_{7}$ effectiveness $*$ wave $3+\beta_{8}$ safety $*$ wave 2

$+\beta_{9}$ safety $*$ wave $3+\beta_{10}$ astrazeneca $/ j \& j *$ effectiveness $+\beta_{11}$ astrazeneca $/ j \& j$

$*$ safety $+\beta_{12}$ astrazeneca $/ j \& j *$ wave $2+\beta_{13}$ astrazeneca $/ j \& j *$ wave 3

$+\beta_{14}$ astrazeneca $/ j \& j *$ effectiveness $*$ wave $2+\beta_{15}$ astrazeneca $/ j \& j *$ effectiveness

$*$ wave $3+\beta_{16}$ astrazeneca $/ j \& j *$ safety $*$ wave $2+\beta_{17}$ astrazeneca $/ j \& j *$ safety

$*$ wave $3+\varepsilon$

Finally, we conduct exploratory analyses to evaluate which groups of citizens exhibit the greatest hesitancy towards the AstraZeneca or Johnson \& Johnson vaccines. We focus on age, vaccination intention, anti-intellectualism or trust in experts, support for childhood vaccination, COVID-19 threat perceptions, and COVID-19 news exposure. We cannot make causal claims as to which specific trait moderates our treatment, but we can gain a general sense of the profile of individuals who are more likely to differentiate between vaccine brands.

We do not have clear theoretical expectations on the direction of the effects. On the one hand, it is possible we may observe stronger effects among groups that are typically more supportive of vaccines. These individuals could be more responsive to communication from public health authorities and experts about the comparative efficacy and safety of COVID-19 vaccines, while vaccine skeptics would be generally unresponsive to communications disseminated by sources they do not trust.

On the other hand, we could observe something akin to a motivated reasoning process, where vaccine skeptics are persuaded by information about the comparatively higher risk and lower efficacy of the AstraZeneca and Johnson \& Johnson vaccines because of its usefulness in bolstering their prior beliefs about the risks of vaccination, while vaccine supporters resist this information because of its perceived threat to their beliefs that COVID-19 vaccines are safe and effective.

We fit a series of linear models including an interaction between these demographic/attitudinal covariates and a binary indicator of AstraZeneca or Johnson \& Johnson treatment ( 1 =AstraZeneca/Johnson \& Johnson, $0=$ Pfizer/Moderna) using OLS:

intention $=\alpha+\beta_{1}$ astrazeneca $/ j \& j+\beta_{2}$ covariate $+\beta_{3}$ astrazeneca $/ j \& j *$ covariate $+\varepsilon$

We describe the measurement of our covariates in Table S2 of the Supplementary Materials.

\section{Results}

Our pre-registered expectations were met when pooling across our three survey waves. Intention is 0.14 points lower on a $0-1$ scale in the AstraZeneca and Johnson \& Johnson conditions compared to Pfizer and Moderna, consistent with H1 (95\% CI $=-0.16,-0.12$; $\mathrm{p}<0.001)$. Perceived effectiveness is 0.11 points lower on a $0-1$ scale in the AstraZeneca and Johnson \& Johnson conditions compared to Pfizer and Moderna, consistent with H2 (95\% CI = -0.12, $-0.09 ; \mathrm{p}<0.001)$. 
Perceived safety is also 0.11 points lower on a 0-1 scale in the AstraZeneca and Johnson \& Johnson conditions compared to Pfizer and Moderna, consistent with H3 (95\% CI = -0.12, -0.09 ; p $<0.001$ ). Our pre-registered expectations were met. There are other, less sizable effects. Hesitancy towards the AstraZeneca vaccine appears to exceed that of Johnson \& Johnson and skepticism of Moderna appears to be slightly higher than Pfizer. These differences are significant at the $\mathrm{p}<0.001$ level and are apparent in the model predictions from equation 1 that are presented in Figure 1.
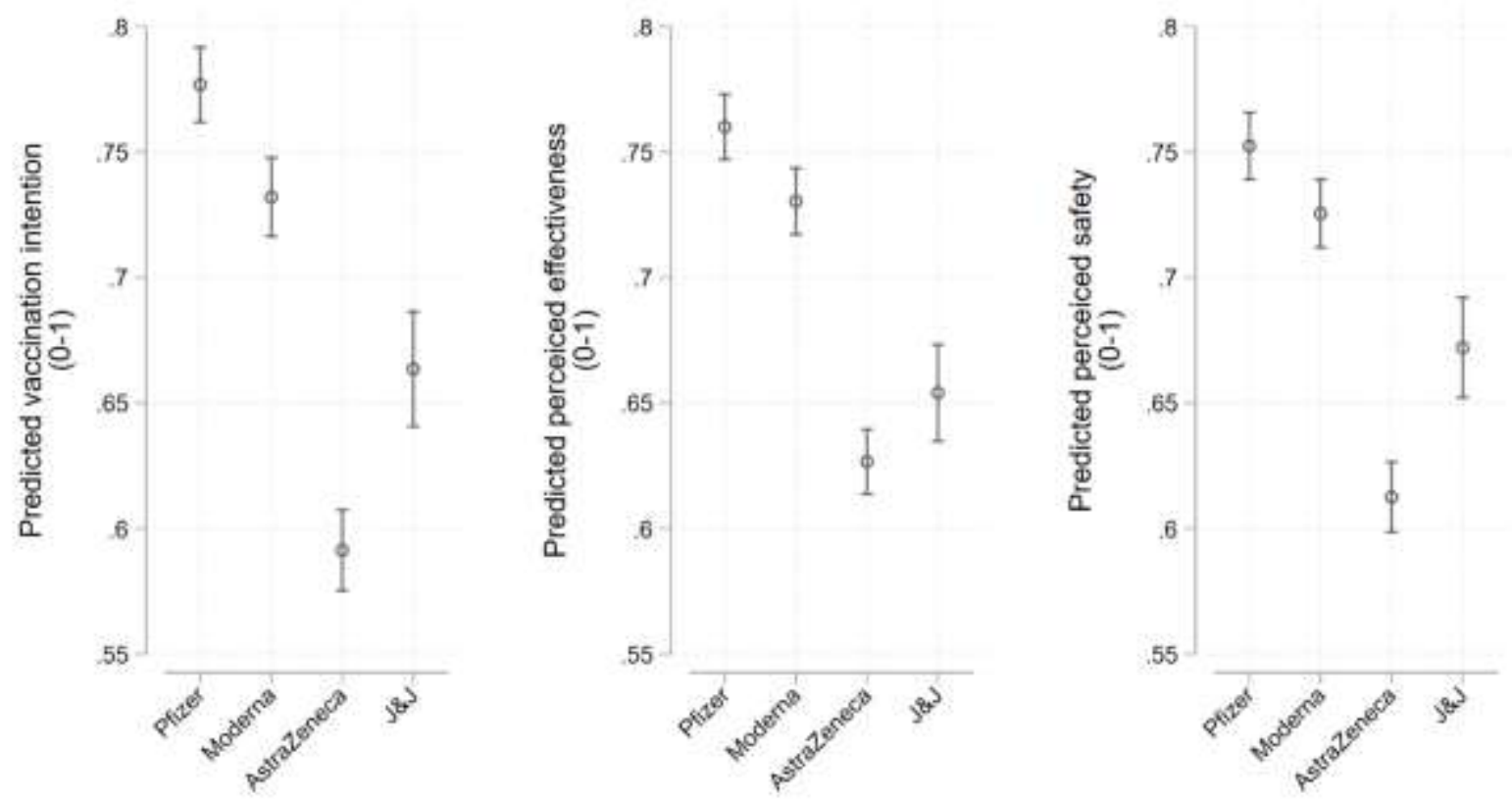

Figure 1. Mean vaccination intention (left), perceived effectiveness (centre), and perceived safety (right) across brand conditions. Results pooled across three waves. Model predictions from equation 1. Estimates can be found in Table S3 of the Supplementary Materials. 95\% confidence intervals.

There are also important differences in people's comparative hesitancy towards available COVID-19 vaccine brands over the time of our fielding. The estimated marginal effects are shown in Figure 2 (from equation 2). In wave 1 were observed only a 0.10 point difference in intention between the Pfizer and AstraZeneca conditions (95\% CI $=-0.13,-0.07$; $\mathrm{p}<0.001$ ). This effect more than doubled to 0.22 points by wave $2(95 \% \mathrm{CI}=-0.26,-0.19 ; \mathrm{p}<0.001)$ and tripled to 0.33 points by wave $3(95 \% \mathrm{CI}=-0.38,-0.28 ; \mathrm{p}<0.001)$. We see a similar pattern with Johnson \& Johnson after the FDA recommended a pause in its administration. Intention was initially 0.09 points lower in the Johnson \& Johnson condition compared to Pfizer in wave $2(95 \% \mathrm{CI}=-0.12,-0.05$; $\mathrm{p}<$ 0.001). This grew to 0.26 points lower by wave 3 after the FDA announcement $(95 \% \mathrm{CI}=-0.31$, $0.21 ; \mathrm{p}<0.001)$. 

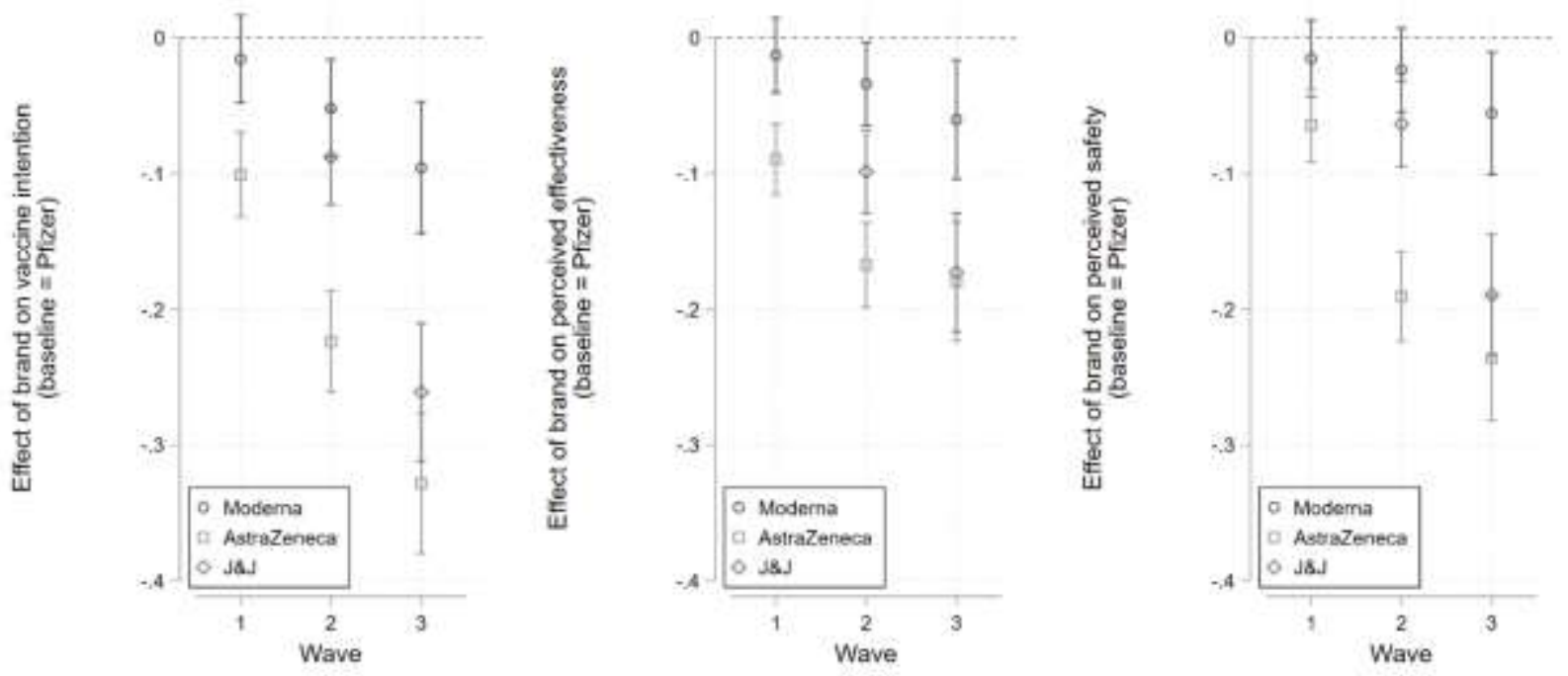

Figure 2. Marginal effect of brand treatment on intention (left), perceived effectiveness (centre), and perceived safety (right) across waves. Reference $=$ Pfizer. Wave $1=$ February 23-March 1, 2021 $(\mathrm{N}=2,495)$; Wave 2 = March 17-23, 2021 ( $\mathrm{N}=2,511)$; Wave 3 = April 15-20, $2021(\mathrm{~N}=1,455) .95 \%$ confidence intervals. Regression estimates from equation 2 can be found in Table S4.

We also see evidence of priming effects: safety perceptions became a more important determinate of intention over the course of fielding. In the top two panels of Figure 3 we display the marginal effects of perceived effectiveness (left) and safety (right) on intention across our three waves (from equation 3). The marginal effect of perceived effectiveness on intention (both on 0-1 scales) dropped from 0.43 in wave $1(95 \% \mathrm{CI}=0.36,0.49, \mathrm{p}<0.001)$ to 0.30 in wave $3(95 \% \mathrm{CI}=$ $0.20,0.38, \mathrm{p}<0.001)$. This difference is statistically significant $(\mathrm{p}=0.019)$. At the same time, the marginal effect of perceived safety increased from 0.57 in wave $1(95 \% \mathrm{CI}=0.51,0.63, \mathrm{p}<0.001)$ to 0.69 in wave $3(95 \% \mathrm{CI}=0.61,0.78, \mathrm{p}<0.001)$. This difference is also statistically significant $(\mathrm{p}$ $=0.017$.

It does not appear, however, that this is true irrespective of the vaccine in question. The centre and bottom panels of Figure 3 plot the marginal effects of perceived effectiveness and perceived safety, respectively, on intention (from equation 4) for those in the Pfizer/Moderna conditions (left) and those in the AstraZeneca/Johnson \& Johnson conditions (right). We only observe a growing (weakening) link between safety (effectiveness) perceptions in the AstraZeneca/Johnson \& Johnson conditions. ${ }^{7}$

\footnotetext{
${ }^{7}$ It also appears that this priming effect occurs exclusively among respondents with higher levels of news consumption. These results can be found in Figures S1 and S2 in the Supplementary Materials.
} 

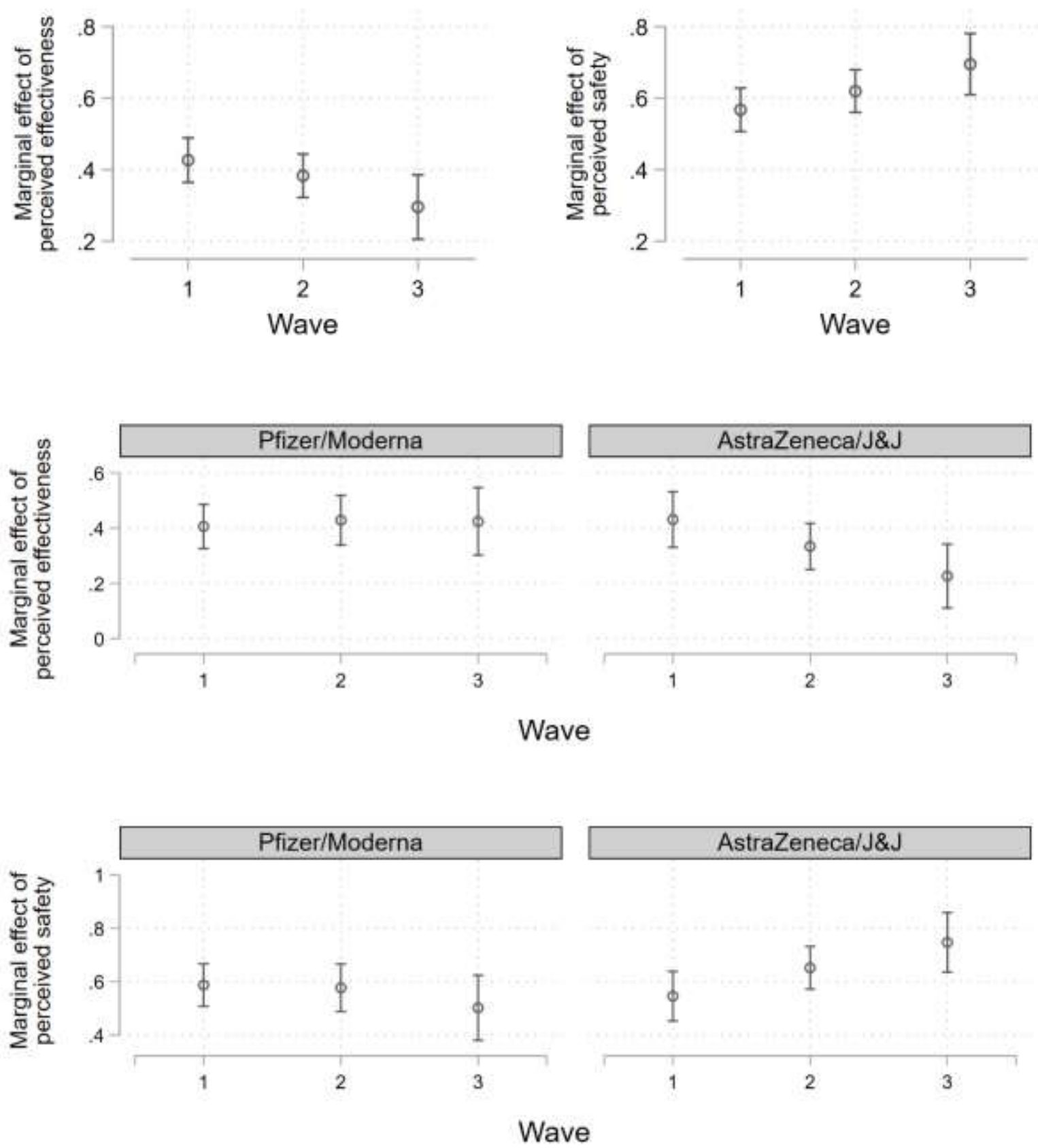

Figure 3. Marginal effect of perceived effectiveness on intention (top-left). Marginal effect of perceived safety on intention (top-right). Marginal effect of perceived effectiveness on Pfizer or Moderna intention (centre-left) and AstraZeneca or Johnson \& Johnson intention (centre-right). Marginal effect of perceived safety on Pfizer or Moderna intention (bottom-left) and AstraZeneca or Johnson \& Johnson intention (bottom-right). Note: 95\% confidence intervals. J\&J= Johnson \& Johnson. Regression estimates derived from equations 3 and 4 can be found in Table S5. 
Our results raise the question of who is most likely to differentiate between COVID-19 brands, especially between Pfizer and Moderna, on the one hand, and AstraZeneca and Johnson \& Johnson, on the other. The marginal effects of the models estimates from equation 5 are shown in Figure 4. Broadly speaking, comparative hesitancy towards the AstraZeneca and Johnson \& Johnson vaccines is not strongest among the typically vaccine hesitant. First, we see stronger effects among older respondents. Intention is estimated to be 0.08 points lower in the AstraZeneca and Johnson \& Johnson conditions compared to Pfizer and Moderna for those aged 24 (95\% CI $=-0.11,-0.05$; $\mathrm{p}<$ 0.001; top-left panel). This difference grows to 0.20 points for those aged $72(95 \% \mathrm{CI}=-0.23,-0.17$; $\mathrm{p}<0.001 ;$ top-left panel).
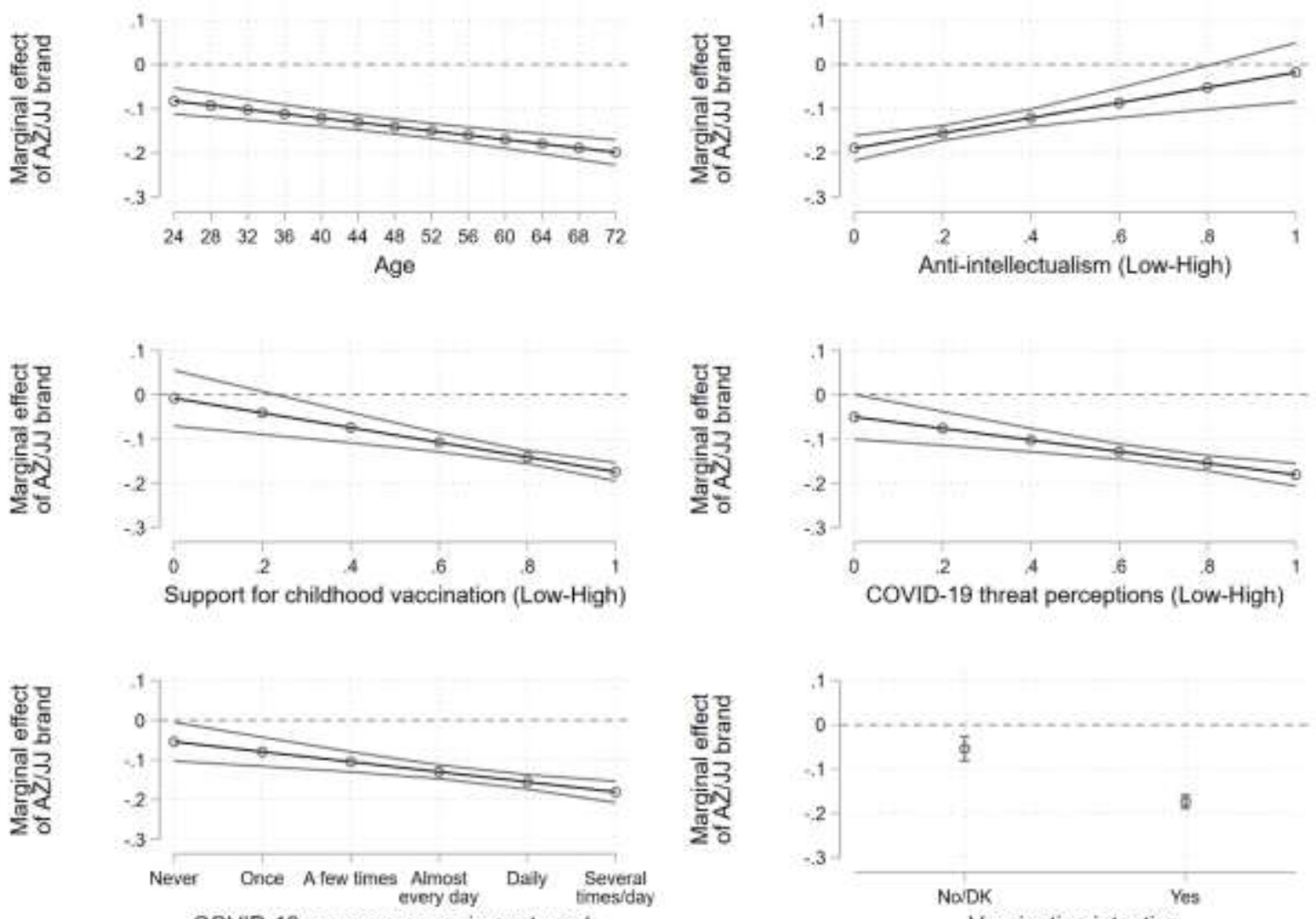

COVID-19 news exposure in past week

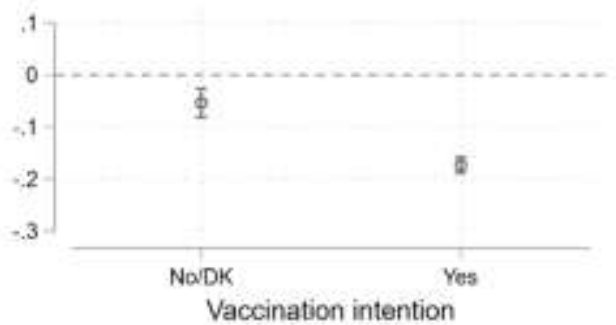

Figure 4. Marginal effect of AstraZeneca or Johnson \& Johnson brand, compared to Pfizer and Moderna, across age (top-left), anti-intellectualism (top-right), support for childhood vaccination (centre-left), COVID-19 threat perceptions (centre-right), COVID-19 news exposure (bottom-left), and previously reported vaccination intention (bottom-right). Note: $95 \%$ confidence intervals. DK $=$ Don't know. AZ = AstraZeneca, JJ= Johnson \& Johnson. Regression estimates from equation 5 can be found in Table S6.

Second, we stronger effects among people with predispositions sympathetic to vaccinations. Among those who are most trusting of experts, we would expect a 0.19 point difference in intention 
between the AstraZeneca and Johnson \& Johnson conditions compared to Pfizer and Moderna (95\% CI $=-0.22,-0.16 ; \mathrm{p}<0.001$; top-right panel). In contrast, we expect a small and not statistically significant 0.02 point difference for those with the highest levels of anti-intellectual sentiment $(95 \% \mathrm{CI}=-0.08,0.05 ; \mathrm{p}=0.595$; top-right panel). For those who are most hostile to childhood vaccinations, we expect only a small and not statistically significant difference in intention between the AstraZeneca and Johnson \& Johnson conditions compared to Pfizer and Moderna (0.01; 95\% CI $=-0.07,0.05 ; \mathrm{p}=0.798$; centre-left panel). Among those who are most supportive of childhood vaccination, the expected difference grows to 0.17 points $(95 \% \mathrm{CI}=-0.19,-0.15 ; \mathrm{p}<$ 0.001 ; centre-left panel).

Third, we see stronger effects among people who perceive the most risk from COVID-19 and among those who are most attentive to COVID-19 news. The difference in intention between the AstraZeneca and Johnson \& Johnson conditions compared to Pfizer and Moderna is 0.18 points for those who perceive the threat from COVID-19 to be most serious $(95 \% \mathrm{CI}=-0.21,-0.16$; $\mathrm{p}<$ 0.001; centre-right panel). This difference weakens to 0.05 points among those who perceive the least amount of threat, which is not quite statistically significant $(95 \% \mathrm{CI}=-0.05,0.00 ; \mathrm{p}=0.051$; centre-right panel). Similarly, the difference in intention between the AstraZeneca and Johnson \& Johnson conditions compared to Pfizer and Moderna is larger among those who pay attention to COVID-19 news several times a day $(-0.18 ; 95 \% \mathrm{CI}=-0.21,-0.15 ; \mathrm{p}<0.001$; bottom-left panel) than those who do not pay attention at all $(-0.05 ; 95 \% \mathrm{CI}=-0.10,-0.01 ; \mathrm{p}=0.030$; bottom-left panel).

Finally, the difference in intention between the AstraZeneca and Johnson \& Johnson conditions compared to Pfizer and Moderna is more than three times as strong among those who reported they would take a COVID-19 vaccine when offered at an earlier stage of the survey $(-0.17$; $95 \% \mathrm{CI}=-0.19,-0.16 ; \mathrm{p}<0.001 ;$ bottom-right panel) than those who said no or expressed uncertainty on this question $(-0.05 ; 95 \% \mathrm{CI}=-0.08,-0.03 ; \mathrm{p}<0.001$; bottom-right panel). Comparative hesitancy towards AstraZeneca and Johnson \& Johnson is strongest among those who are most amenable to vaccination.

\section{Discussion}

Mass COVID-19 vaccination campaigns have to wrestle with the challenges posed by vaccine hesitancy. One dimension of this challenge is quite unlike that for other more established vaccines: there are multiple vaccines with different safety and efficacy profiles with levels of availability and accessibility that vary over time and across national contexts. People may habour strong preferences for, or aversion to, specific vaccines. This can pose a problem for public health agencies relying on less-preferred vaccines.

Our analysis provide three central contributions. First, we show that there is a significant level of aversion to COVID-19 vaccines from AstraZeneca and Johnson \& Johnson in the Canadian context, consistent with our pre-registered expectations. Descriptively, it appears that our brand 
treatments mostly influenced willingness to take the randomly assigned vaccine indirectly through safety and efficacy perceptions (see Supplementary Materials).

Second, we illustrate important dynamics in this process by leveraging the time period of our analysis. Hesitancy towards the AstraZeneca and Johnson \& Johnson vaccines intensified greatly as public health agencies in Canada and the U.S. issued warnings and restrictions on their usage and news media covered these developments. These communications also appear to have shifted the fundamental drivers of people's willingness to take certain vaccines. Perceptions of vaccine safety grew in importance in explaining willingness to take a given vaccine if offered, particularly among heavy news consumers in the AstraZeneca and Johnson \& Johnson treatment conditions. As the communication environment highlighted safety concerns around these vaccines, attentive citizens responded accordingly.

Importantly, hesitancy towards the AstraZeneca and Johnson \& Johnson vaccines did not emerge solely - or even primarily - due to actions by domestic health officials. For example, although Johnson \& Johnson had been approved by Health Canada, it had not been administered to Canadians and Canadian public health agencies had not changed their usage recommendations. We observe increased aversion towards Johnson \& Johnson entirely due to decisions made by foreign regulatory agencies and related news coverage.

Third, we show that it is not the case that vaccine hesitant groups are more likely to be repelled by the AstraZeneca and Johnson \& Johnson vaccines as we might expect if people engaged in motivated reasoning - accepting and rejecting communication related to the safety and efficacy of these vaccines based on its convenience for their prior beliefs about COVID-19 vaccines more generally. Instead, we find that individuals who are more trusting of experts, more supportive of childhood vaccines, and wearier of the threat posed by COVID-19 are more inclined to differentiate by brand at the expense of AstraZeneca and Johnson \& Johnson. ${ }^{8}$ We likely cannot tackle vaccinespecific hesitancy with communication strategies aimed at those skeptical of vaccines and scientific expertise generally.

Our results occur in a national context (Canada) where several vaccines have been approved, but are not equally available to all citizens. It is an open question whether comparative hesitancy towards certain vaccines would exist absent the potential of receiving another vaccine, or if they are endogenous to a supply of more than one vaccine. It is also an open question, given skepticism of specific vaccines, whether countries may actually slow down vaccination rates by procuring multiple types of vaccine, only some of which citizens will be willing to take if the potential of waiting for another vaccine exists. Importantly, we find little evidence that overall intention to vaccinate dwindled as controversy swirled about the AstraZeneca and Johnson \& Johnson vaccines. The share

\footnotetext{
${ }^{8}$ A more mechanical explanation for this result could be floor effects, where vaccine hesitant groups are so resistant to COVID-19 vaccines that there is little room for the values of outcome variables to fall further in response to random assignment. In Table S7 we provide the baseline values for vaccination intention for high and low values of each of our examined covariates and by vaccine condition. Although we cannot rule out floor effects, it appears quite unlikely.
} 
of citizens reporting that they intend to vaccinate (or already had) increased 4 points by wave 2 (95\% $\mathrm{CI}=0.01,0.06, \mathrm{p}=0.003)$ and 9 points by wave $3(95 \% \mathrm{CI}=0.06,0.12, \mathrm{p}<0.001)$.

Nevertheless these findings are still important. It is clear that people are not solely responsive to information from domestic health authorities. Communications and recommendations by foreign health agencies - at least when paired with substantial news coverage - are also highly influential. There is a risk that attentive individuals in countries with limited supply and a less diverse portfolio of vaccines may turn against the few vaccines that happen to be available depending on these dynamics. This is perhaps most threatening with AstraZeneca, which is logistically much more attractive to developing countries than mRNA vaccines. More cross-national research is needed to observe whether these dynamics hold in contexts with less volume and diversity in vaccine supply.

The importance of these findings is also apparent looking forward. As variants of COVID-19 continue to emerge, governments will need to distribute additional booster shots. This puts added stress on vaccine supply. People may be less able to get their preferred vaccines immediately and could respond by holding out until their preferred vaccine becomes available. It appears that at the time of our study Canadians were willing to wait a considerable amount of time to avoid taking the AstraZeneca or Johnson \& Johnson vaccines. In the Supplementary Materials we provide evidence from two additional surveys where we ask respondents for their preferred vaccine and ask them how long they would be willing to wait for it, rather than an immediately available, randomly assigned alternative (like AstraZeneca). Only $23 \%$ of respondents reported a willingness to take AstraZeneca or Johnson \& Johnson immediately, 41\% were willing to wait 12 months or more for their preferred vaccine, while $36 \%$ were willing to tolerate some intermediate delay.

It is also possible that the set of available vaccines will change in Canada and elsewhere in the future. Variants may emerge that successfully evade the protection of currently available vaccines and require new products to be developed. And, of course, COVID-19 will not be the last pandemic we encounter. In either case, we may again see competing vaccines with important variation in their safety and efficacy profiles, as well as their availability, that together produce vaccine-specific hesitancy in different national contexts. We need more attentiveness to these dynamics. They may prove to be immensely consequential in situations where there is some degree of vaccine choice, but relatively limited supply that prevents people from getting the vaccines they prefer.

There are some important limitations to our study. We evaluate behavioural intention and attitudes as opposed to observed behaviour. We cannot make strong claims that self-reported aversion to AstraZeneca or Johnson \& Johnson is indicative of respondents' behaviour and this is especially true considering the limited distribution of these vaccines in Canada. Further research should be conducted in countries more dependent on these vaccines for the success of their mass vaccination campaigns. Notwithstanding our inability to link attitudes and behavioural intention to observed behaviour, our results consistently demonstrate the existence of vaccine-specific hesitancy and a particular aversion to those offered by AstraZeneca.

Acknowledging the existence of vaccine-specific hesitancy raises questions of how to combat it. Our findings suggest we cannot rely on communication strategies that target those that are 
generally skeptical of vaccines. We encourage future research on alternative communication strategies that can enhance uptake of less-preferred vaccines.

\section{References}

Advisory Committee on Immunization Practices. (2021). Overview of Janssen's Single-Dose COVID-19 Vaccine, Ad26.COV2.S. US Centers for Disease Control and Prevention. February 28. https://www.cdc.gov/vaccines/acip/meetings/downloads/slides-2021-02/2803-01/02-COVID-Douoguih.pdf.

Allcott, H., Boxell, L., Conway, J., Gentzkow, M., Thaler, M., \& Yang, D. (2020). Polarization and public health: Partisan differences in social distancing during the coronavirus pandemic. Journal of Public Economics, 191, 104254. doi: 10.1016/j.jpubeco.2020.104254.

Baumgaertner, B., Carlisle, J.E., \& Justwan, F. (2018). The influence of political ideology and trust on willingness to vaccinate. PloS One, 13(1), e0191728. doi: 10.1371/journal.pone.0191728.

Bullock, J. G., Green, D. P., \& Ha, S. E. (2010). Yes, but what's the mechanism? (Don't expect an easy answer). Journal of Personality and Social Psychology, 98(4), 550-558. doi: 10.1037/a0018933.

Callaghan, T., Moghtaderi, A., Lueck, J.A., Hotez, P., Strych, U., Dor, A., et al. (2021). Correlates and disparities of intention to vaccinate against COVID-19. Social Science \& Medicine, 252, 113638. doi: 10.1016/j.socscimed.2020.113638.

Elkin, L.E, Pullon, S.R.H., \& Stubbe, M.H. (2020). Should I vaccinate my child? Comparing the displayed stances of vaccine information retrieved from Google, Facebook and YouTube. Vaccine, 38(13), 2771-2778

Fowler, E.F., \& Gollust, S.E. (2015). The content and effect of politicized health controversies. Annals of the American Academy of Political and Social Science, 658(1), 155-171.

Gadarian, S.K., Goodman, S.W., Pepinsky, T.B. (2021). Partisanship, health behavior, and policy attitudes in the early stages of the COVID-19 pandemic. PloS One, 16(4), e0249596. doi: 10.1371/journal.pone.0249596.

Jamison, A.M, Broniatowski, D.A, Dredze, M., Wood-Doughty, Z., Khan, D., Quinn, S.C. (2020). Vaccine-related advertising in the Facebook ad archive. Vaccine, 38(3), 512-520.

Joslyn, M.R., \& Sylvester, S.M. (2019). The determinants and consequences of accurate beliefs about childhood vaccinations. American Politics Research, 47(3), 628-649.

Kaplan, R., \& Milstein, A. (2021). Influence of a COVID-19 vaccine's effectiveness and safety profile on vaccination acceptance. Proceedings of the National Academu of Sciences, 118(10), e2021726118. doi: 10.1073/pnas.2021726118. 
Keelan, J., Pavri-Garcia, V., Tomlinson, G., \& Wilson, K. (2007). YouTube as a source of information on immunization: a content analysis. Journal of the American Medical Association, 298(21), 2482-2484.

Kreps, S., Prasad, S., Brownstein, J.S., Hswen, Y., Garibaldi, B.T., Zhang, B., et al. (2020). Factors associated with us adults' likelihood of accepting COVID-19 vaccination. JAMA Network. Open., 3(10), e2025594. doi: 10.1001/jamanetworkopen.2020.25594.

Merkley, E., \& Loewen, P.J. (2021). Anti-intellectualism and the mass public's response to the COVID-19 pandemic. Nature Human Behaviour, 5, 706-715. doi:10.1038/s41562-021-01112-w.

Merkley, E. \& Loewen, P.J. (In press). Assessment of Communication Strategies for Mitigating COVID-19 Vaccine-Specific Hesitancy in Canada. JAMA Network Open.

Motta, M. (2020). President Trump promised a COVID vaccine by Election Day: That politicized vaccination intentions. In D. Jackson, D.S. Coombs, F. Trevisan, D. Lilleker, \& E. Thorse (eds.), U.S. election analysis 2020: Media, voters and the campaign (pp. 18-19). Poole, U.K.: The Centre for Comparative Politics and Media Research, Bournemouth University.

Motta, M. (2021a). Can a COVID-19 vaccine live up to Americans' expectations? A conjoint analysis of how vaccine characteristics influence vaccination intentions. Social Science \& Medicine, 272, 113642. doi: 10.1016/j.socscimed.2020.

Motta, M. (2021b). Republicans, not Democrats, are more likely to endorse anti-vaccine misinformation. American Politics Research, 49(5), 428-438. https://doi.org/10.1177/1532673X211022639

Motta, M., Callaghan, T., \& Sylvester, S. (2018). Knowing less but presuming more: DunningKruger effects and the endorsement of anti-vaccine policy attitudes. Social Science \& Medicine, 211, 274-281. https://doi.org/10.1016/j.socscimed.2018.06.032.

Pennycook, G., McPhetres, J., Bago, B., \& Rand, D.G. (2021). Beliefs about COVID-19 in Canada, the United Kingdom, and the United States: A novel test of political polarization and motivated reasoning. Personality and Social Psychology Bulletin. doi:10.31234/osf.io/zhjkp.

Shah, Z., Surian, D., Dyda, A., Coiera, E., Mandl, K.D, \& Dunn A.G. (2019). Automatically appraising the credibility of vaccine-related web pages shared on social media: a Twitter surveillance study. Journal of Medical Internet Research, 21 (11), e14007.

Stecula, D.A., Kuru, O., \& Jamieson, K.H. (2020). How trust in experts and media use affect acceptance of common anti-vaccination claims. Havard Kennedy School Misinformation Review. doi: $10.37016 / \mathrm{mr}-2020-007$.

Tang, L., Fujimoto, K., Amith, M., Cunningham, R., Costantini, R., York, F., Xiong, G., Boom, J., \& Tao, C. (2021). Down the rabbit hole of vaccine misinformation on YouTube: Network exposure study. Journal of Medical Internet Research, 23(1), e23262. 
Teo, S.P. (2021). Review of COVID-19 vaccines and their evidence in older adults. Annals of Geriatric Medicine Research, 25(1), 4-9.

Wolfe, R.M., \& Sharpe, L.K. (2002). Anti-vaccinationists past and present. British Medical Journal, 325, 430-432. 


\section{Supplementary Materials}

The correlates and dynamics of COVID-19 vaccine-specific hesitancy

\section{Contents}

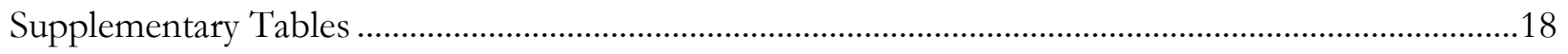

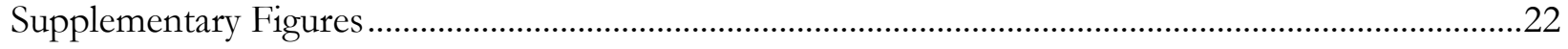

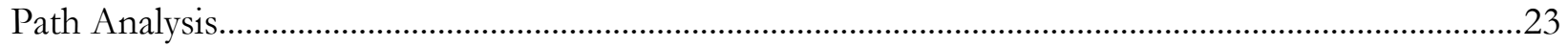

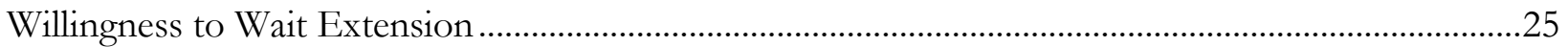




\section{Supplementary Tables}

Table S1. Demographic Breakdown of Sample

\begin{tabular}{lccccc}
\hline & Census & Pooled & Wave 1 & Wave 2 & Wave 3 \\
\hline Female & 51.4 & 52.0 & 51.2 & 53.1 & 51.6 \\
$18-34$ & 27.3 & 26.4 & 27.1 & 25.8 & 26.1 \\
$34-54$ & 34.1 & 34.1 & 33.6 & 34.5 & 34.4 \\
$55+$ & 38.6 & 39.5 & 39.3 & 39.8 & 39.4 \\
French & 21.4 & 20.2 & 20.8 & 19.2 & 21 \\
Atlantic & 6.8 & 6.6 & 6.8 & 6.8 & 6.1 \\
Quebec & 23.4 & 23.2 & 23.5 & 22.6 & 23.8 \\
Ontario & 38.3 & 38.4 & 38.1 & 38.7 & 38.6 \\
West & 31.2 & 31.7 & 31.5 & 31.9 & 31.6 \\
\hline
\end{tabular}

Table S2. Covariate Measurement

\begin{tabular}{ll}
\hline Covariate & Measurement \\
\hline $\begin{array}{l}\text { COVID-19 Vaccination } \\
\text { Intention }\end{array}$ & Would take a COVID-19 vaccine if available (Yes, No, Unsure) \\
\hline & $\begin{array}{l}\text { Trust in the following groups: experts, doctors, medical } \\
\text { professionals, scientists, university professors, economists } \\
\text { (distrust a lot, distrust somewhat, neither trust nor distrust, trust } \\
\text { Anti-intellectualism }\end{array}$ \\
& somewhat, trust a lot; 0-1 index) \\
\hline
\end{tabular}

Level of agreement or disagreement with the following statements: 1) Childhood vaccines are important for the health of children; 2) Getting vaccines is a good way to protect children from disease; 3) Having children vaccinated is

Support for Childhood Vaccination important for the health of others in my community; 4) All childhood vaccines offered by the government in my community are beneficial; 5) The information I receive about vaccines from the government is reliable and trustworthy; 6) Generally I do what my doctor or health care provider recommends about vaccines (strongly agree/disagree, somewhat agree/disagree, or neither agree nor disagree; 0-1 index)

How serious of a threat is COVID-19 to 1) yourself; 2) other

COVID-19 Risk Perceptions Canadians? (very serious, somewhat serious, not very serious, not at all serious; $0-1$ index)

How often in the past week have you read, watched, or listened COVID-19 News Exposure to news related to the COVID-19 pandemic (never, once, a few times, almost every day, daily, and several times a day) 
Table S3. Figure 1 Coefficient Estimates

\begin{tabular}{lcccccc}
\hline & \multicolumn{2}{c}{ Intention } & \multicolumn{2}{c}{ Effectiveness } & \multicolumn{2}{c}{ Safety } \\
& Coef. & SE & Coef. & SE & Coef. & SE \\
\hline Moderna & $-0.04^{* * *}$ & 0.01 & $-0.03^{* * *}$ & 0.01 & $-0.03^{* * *}$ & 0.01 \\
AstraZeneca & $-0.19^{* * *}$ & 0.01 & $-0.13^{* * *}$ & 0.01 & $-0.14^{* * *}$ & 0.01 \\
J\&J (Wave 2 and 3) & $-0.11^{* * *}$ & 0.01 & $-0.11^{* * *}$ & 0.01 & $-0.08^{* * *}$ & 0.01 \\
Wave 2 & 0.00 & 0.01 & $0.02^{* * *}$ & 0.01 & 0.01 & 0.01 \\
Wave 3 & $-0.04^{* * *}$ & 0.01 & 0.01 & 0.01 & $-0.03^{* * *}$ & 0.01 \\
Constant & $0.78^{* * *}$ & 0.01 & $0.75^{* * *}$ & 0.01 & $0.75^{* * *}$ & 0.01 \\
\hline $\mathrm{R}$ & 0.05 & \multicolumn{3}{c}{0.04} & & 0.04 \\
$\mathrm{~N}$ & 6248 & \multicolumn{6}{c}{6248} & & 6248 \\
\hline
\end{tabular}

HC2 robust standard errors; ${ }^{*} \mathrm{p}<0.1,{ }^{* *} \mathrm{p}<0.05,{ }^{* * *} \mathrm{p}<0.01$. Note: J\&J $=$ Johnson \& Johnson treatment.

Table S4. Figure 2 Coefficient Estimates

\begin{tabular}{|c|c|c|c|c|c|c|}
\hline & \multicolumn{2}{|c|}{ Intention } & \multicolumn{2}{|c|}{ Effectiveness } & \multicolumn{2}{|c|}{ Safety } \\
\hline & Coef. & SE & Coef. & SE & Coef. & $\mathrm{SE}$ \\
\hline Moderna & -0.02 & 0.02 & -0.01 & 0.01 & -0.02 & 0.01 \\
\hline AstraZeneca & $-0.10 * * *$ & 0.02 & $-0.09 * * *$ & 0.01 & $-0.06 * * *$ & 0.01 \\
\hline J\&J (Wave 2) & $-0.26^{* * *}$ & 0.03 & $-0.17 * * *$ & 0.02 & $-0.19 * * *$ & 0.02 \\
\hline Wave 2 & $0.05^{* * *}$ & 0.02 & $0.05^{* * *}$ & 0.01 & $0.05^{* * *}$ & 0.02 \\
\hline Wave 3 & $0.08 * * *$ & 0.02 & $0.06^{* * *}$ & 0.02 & $0.06 * * *$ & 0.02 \\
\hline Moderna $*$ Wave 2 & -0.04 & 0.02 & -0.02 & 0.02 & -0.01 & 0.02 \\
\hline Moderna * Wave 3 & $-0.08 * * *$ & 0.03 & $-0.04 *$ & 0.03 & -0.04 & 0.03 \\
\hline AstraZeneca * Wave 2 & $-0.12^{* * *}$ & 0.02 & $-0.08^{* * *}$ & 0.02 & $-0.13^{* * *}$ & 0.02 \\
\hline AstraZeneca * Wave 3 & $-0.23^{* * *}$ & 0.03 & $-0.09 * * *$ & 0.03 & $-0.17 * * *$ & 0.03 \\
\hline J\&J * Wave 3 & $0.17 * * *$ & 0.03 & $0.07 * * *$ & 0.03 & $0.13^{* * *}$ & 0.03 \\
\hline Constant & $0.74 * * *$ & 0.01 & $0.73^{* * *}$ & 0.01 & $0.72 * * *$ & 0.01 \\
\hline $\mathrm{R}^{2}$ & \multicolumn{2}{|c|}{0.06} & \multicolumn{2}{|c|}{0.04} & \multicolumn{2}{|c|}{0.05} \\
\hline $\mathrm{N}$ & \multicolumn{2}{|c|}{6248} & \multicolumn{2}{|c|}{6248} & \multicolumn{2}{|c|}{6248} \\
\hline
\end{tabular}

HC2 robust standard errors; ${ }^{*} \mathrm{p}<0.1,{ }^{* *} \mathrm{p}<0.05,{ }^{* * *} \mathrm{p}<0.01$. Note: $\mathrm{J} \& \mathrm{~J}=$ Johnson $\&$ Johnson treatment. 
Table S5. Figure 3 Coefficient Estimates

\begin{tabular}{|c|c|c|c|c|}
\hline & Coef. & $\mathrm{SE}$ & Coef. & $\mathrm{SE}$ \\
\hline Effectiveness & $0.43^{* * *}$ & 0.03 & $0.41 * * *$ & 0.04 \\
\hline Safety & $0.57 * * *$ & 0.03 & $0.59 * * *$ & 0.04 \\
\hline Wave 2 & -0.01 & 0.01 & -0.02 & 0.02 \\
\hline Wave 3 & -0.02 & 0.02 & $0.06^{* *}$ & 0.03 \\
\hline Wave $2 *$ Effectiveness & -0.04 & 0.04 & 0.02 & 0.06 \\
\hline Wave $3 *$ Effectiveness & $-0.13 * *$ & 0.06 & 0.02 & 0.07 \\
\hline Wave $2 *$ Safety & 0.05 & 0.04 & -0.01 & 0.06 \\
\hline Wave $3 *$ Safety & $0.13^{* *}$ & 0.05 & -0.08 & 0.07 \\
\hline AZ/J\&J & & & -0.01 & 0.02 \\
\hline AZ/J\&J * Effectiveness & & & 0.03 & 0.07 \\
\hline AZ/J\&J $*$ Safety & & & -0.04 & 0.06 \\
\hline AZ/J\&J * Wave 2 & & & 0.01 & 0.03 \\
\hline AZ/J\&J * Wave 3 & & & $-0.10 * * *$ & 0.03 \\
\hline AZ/J\&J * Effectiveness $*$ Wave 2 & & & -0.12 & 0.09 \\
\hline AZ/J\&J $*$ Effectiveness $*$ Wave 3 & & & $-0.22 * *$ & 0.11 \\
\hline AZ/J\&J * Safety $*$ Wave 2 & & & 0.12 & 0.09 \\
\hline AZ/J\&J * Safety $*$ Wave 3 & & & $0.29 * * *$ & 0.10 \\
\hline Constant & 0.01 & 0.01 & $0.02 *$ & 0.01 \\
\hline $\mathrm{R}^{2}$ & \multicolumn{2}{|c|}{0.67} & \multicolumn{2}{|c|}{0.68} \\
\hline $\mathrm{N}$ & \multicolumn{2}{|c|}{6248} & \multicolumn{2}{|c|}{6248} \\
\hline
\end{tabular}

Table S6. Figure 4 Coefficient Estimates

\begin{tabular}{lcccccc}
\hline Covariate & Age & $\begin{array}{c}\text { Anti- } \\
\text { intellectualism }\end{array}$ & $\begin{array}{c}\text { Vaccine } \\
\text { support }\end{array}$ & $\begin{array}{c}\text { COVID-19 } \\
\text { Threat }\end{array}$ & $\begin{array}{c}\text { COVID-19 } \\
\text { News }\end{array}$ & $\begin{array}{c}\text { Vaccine } \\
\text { Intention }\end{array}$ \\
\hline AZ/J\&J & -0.02 & $-0.19^{* * *}$ & -0.01 & $-0.05^{*}$ & $-0.05^{* *}$ & $-0.05^{* * *}$ \\
& $(0.03)$ & $(0.01)$ & $(0.03)$ & $(0.03)$ & $(0.02)$ & $(0.01)$ \\
Covariate & $0.00^{* * *}$ & $-0.66^{* * *}$ & $0.83^{* * *}$ & $0.49^{* * *}$ & $0.07 * * *$ & $0.51^{* * *}$ \\
& $(0.00)$ & $(0.03)$ & $(0.02)$ & $(0.02)$ & $(0.00)$ & $(0.01)$ \\
$\mathrm{AZ} / \mathrm{J} \& \mathrm{~J} *$ Covariate & $-0.00^{* * *}$ & $0.17^{* * *}$ & $-0.17 * * *$ & $-0.13^{* * *}$ & $-0.03^{* * *}$ & $-0.12^{* * *}$ \\
& $(0.00)$ & $(0.04)$ & $(0.04)$ & $(0.03)$ & $(0.01)$ & $(0.02)$ \\
Constant & $0.53^{* * *}$ & $0.95^{* * *}$ & $0.11^{* * *}$ & $0.42^{* * *}$ & $0.52^{* * *}$ & $0.40^{* * *}$ \\
\hline $\mathrm{R}^{2}$ & 0.08 & 0.16 & 0.28 & 0.15 & 0.09 & 0.42 \\
$\mathrm{~N}$ & 6247 & 6248 & 6248 & 6248 & 6248 & 6248 \\
\hline HC2 robust standard errors in parentheses, ${ }^{*} \mathrm{p}<0.1, * * \mathrm{p}<0.05, * * * \mathrm{p}<0.01$. & Note: AZ/J\&J $=$ AstraZeneca or Johnson \\
\& Johnson treatment. & & & & &
\end{tabular}


Table S7. Baseline Vaccination Intention by Covariate Values and Randomly Assigned Vaccine

\begin{tabular}{lcccc}
\hline & \multicolumn{2}{c}{ Pfizer } & \multicolumn{2}{c}{ Moderna } \\
& High & Low & High & Low \\
\hline Age & 0.86 & 0.68 & 0.82 & 0.63 \\
Anti-intellectualism & 0.57 & 0.91 & 0.52 & 0.88 \\
Support for Childhood Vaccines & 0.96 & 0.48 & 0.94 & 0.42 \\
COVID-19 Risk Perceptions & 0.86 & 0.51 & 0.84 & 0.40 \\
COVID-19 News Consumption & 0.84 & 0.63 & 0.79 & 0.58 \\
Vaccination Intention & 0.92 & 0.42 & 0.89 & 0.38 \\
\hline & AstraZeneca & Johnson \& Johnson \\
& High & Low & High & Low \\
\hline Age & 0.63 & 0.56 & 0.72 & 0.60 \\
Anti-intellectualism & 0.44 & 0.70 & 0.47 & 0.79 \\
Support for Childhood Vaccines & 0.83 & 0.40 & 0.86 & 0.41 \\
COVID-19 Risk Perceptions & 0.66 & 0.36 & 0.69 & 0.38 \\
COVID-19 News Consumption & 0.66 & 0.52 & 0.71 & 0.58 \\
Vaccination Intention & 0.72 & 0.33 & 0.77 & 0.37 \\
\hline
\end{tabular}

Note: vaccination intention measured on 0-1 scale. Low and high values based on lowest and highest decile in distribution of covariate with exception of age, which is 18/34 vs. 55 and over. 


\section{Supplementary Figures}

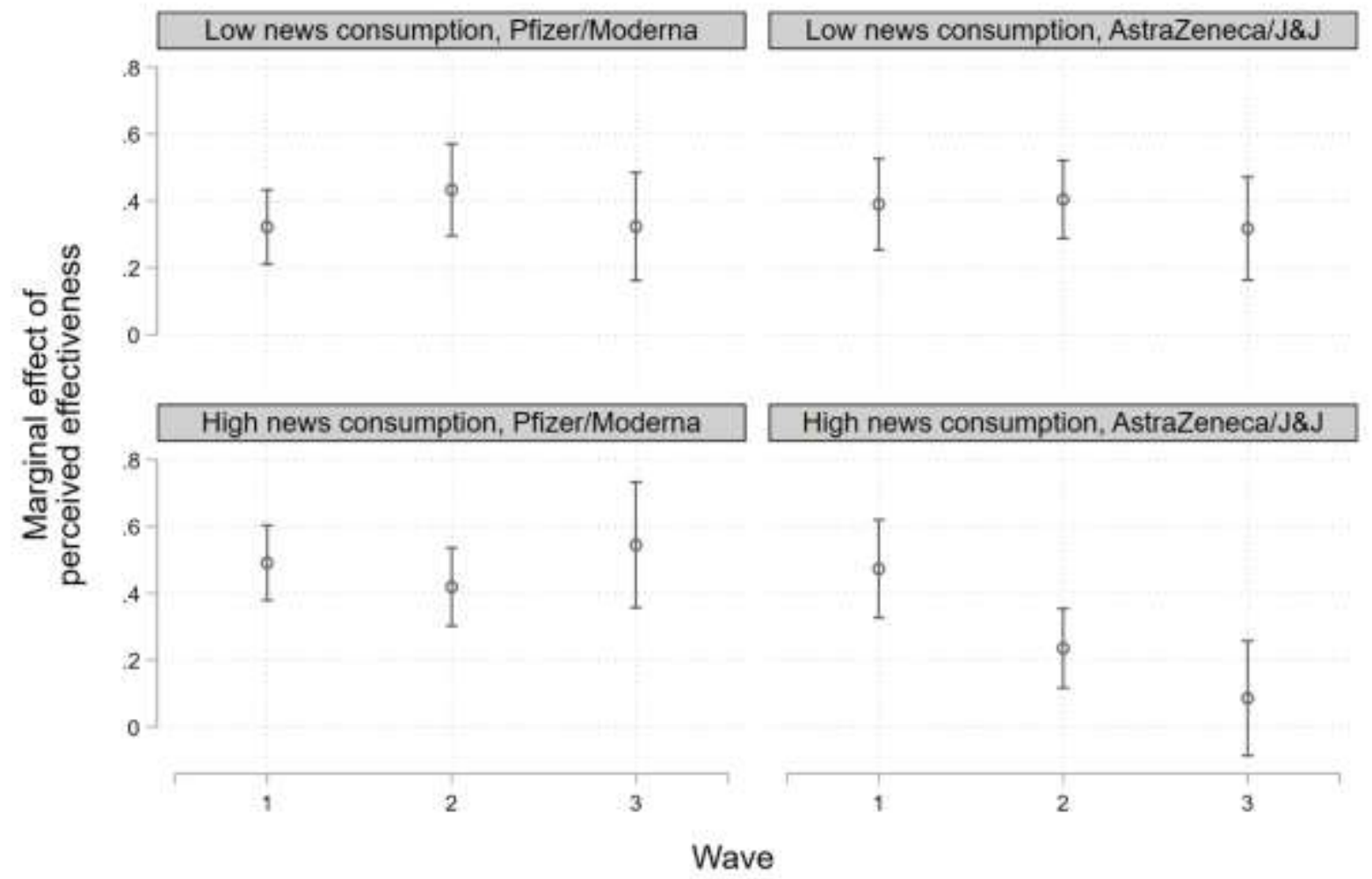

Figure S1. Marginal effect of perceived effectiveness on intention for low- (top) and high-volume (bottom) news consumers in the Pfizer/Moderna (left) or the AstraZeneca/Johnson \& Johnson treatment conditions (right). Note: 95\% confidence intervals. J\&J= Johnson \& Johnson. 


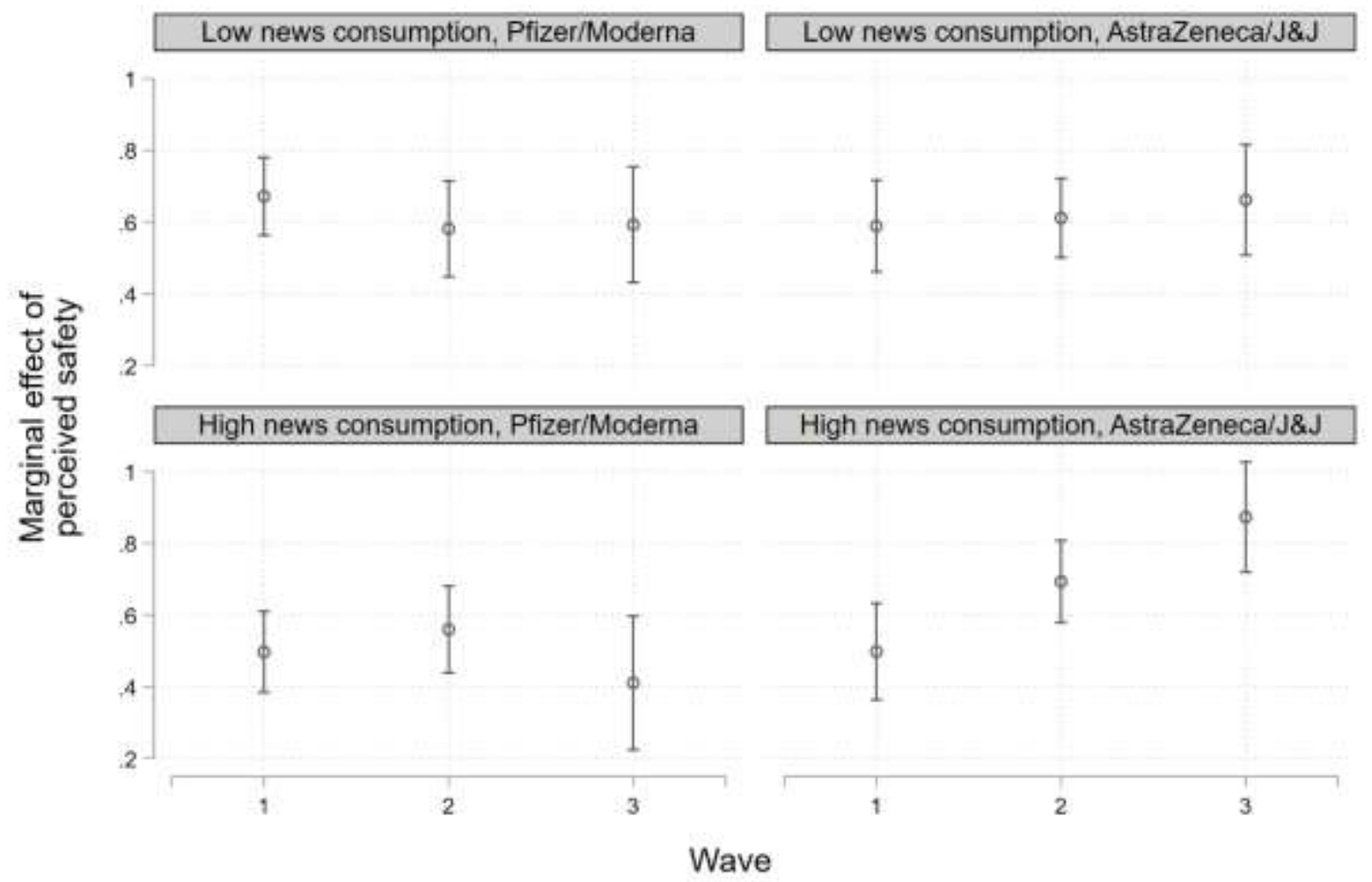

Figure S2. Marginal effect of perceived safety on intention for low- (top) and high-volume (bottom) news consumers in the Pfizer/Moderna (left) or the AstraZeneca/Johnson \& Johnson treatment conditions (right). Note: 95\% confidence intervals. J\&J= Johnson \& Johnson.

\section{Path Analysis}

Our brand treatment likely affects willingness to take the randomized vaccine by affecting their safety and efficacy evaluations. The communication environment provided information to respondents on the comparative safety and efficacy of the AstraZeneca and Johnson \& Johnson vaccines compared to those from Pfizer and Moderna. Random assignment of one vaccine over another will trigger those evaluations and influence their intention. Safety and effectiveness evaluations are therefore a mediating variable between our treatment and outcome.

One common approach to estimate mediations effects is through a path analysis that allows us to tease out the direct influence of our treatment on the outcome from the indirect effects that flow through the mediating variables. This method is extremely limited in its ability to identify causal effects, even with a randomly assigned treatment. It makes strong assumptions that the outcome variable does not affect the mediators in response to treatment, that there aren't other unmeasured mediators that are affected by the treatment are correlated with the observed mediators, and that there is no measurement error (see Bullock et al. (2010) for a more in-depth treatment). 
In this context, for example, it may be possible that people have comparative hesitancy to receive the AstraZeneca and Johnson \& Johnson vaccines, which they rationalize by forming opinions on their safety and effectiveness (i.e., $X \rightarrow Y \rightarrow M$ ). Or, the negativity surrounding AstraZeneca and Johnson \& Johnson may have caused respondents to hold negatively charged feelings towards these vaccines - an alternative mediator that is correlated with safety and efficacy perceptions. A better approach - though not without its own caveats - is to randomly assign mediating variables as well. We did not believe this was feasible for our purposes: randomizing brand as well as safety and efficacy information would entail a significant and potentially harmful deception.

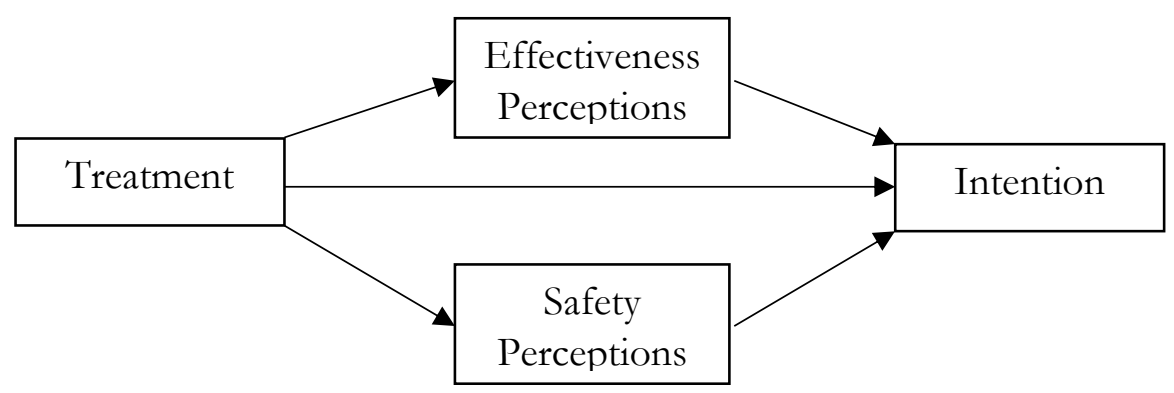

Figure S3. Path Diagram

As a result, we conducted a path analysis using the SEM package in Stata version 16 by estimating the model in Figure S3 where the randomized brand treatment could affect intention directly or indirectly through safety and effectiveness perceptions. We allowed for covariation between safety and effectiveness perceptions. The estimates from the path analysis can be found in Table S8. The treatment is associated with a 0.14 total decrease in intention (on a $0-1$ scale) pooling across all three waves $(95 \% \mathrm{CI}=-0.16,-0.12 ; \mathrm{p}<0.001)$. As expected, over $75 \%$ of this effect is felt indirectly through safety and effectiveness perceptions $(-0.11 ; 95 \% \mathrm{CI}=-0.12,-0.09 ; \mathrm{p}<0.001)$.

Table S8. Path Analysis Estimates

\begin{tabular}{lccccc}
\hline & Coef. & SE & p-value & $95 \%$ LB & $95 \%$ UB \\
\hline AZ/J\&J, Effectiveness & -0.107 & 0.007 & 0.000 & -0.121 & -0.093 \\
AZ/J\&J, Safety & -0.107 & 0.007 & 0.000 & -0.122 & -0.093 \\
Effectiveness, Intention & 0.374 & 0.020 & 0.000 & 0.335 & 0.413 \\
Safety, Intention & 0.612 & 0.019 & 0.000 & 0.574 & 0.650 \\
AZ/J\&J, Intention (Direct) & -0.035 & 0.005 & 0.000 & -0.045 & -0.025 \\
AZ/J\&J, Intention (Indirect) & -0.106 & 0.007 & 0.000 & -0.119 & -0.092 \\
AZ/J\&J, Intention (Total) & -0.141 & 0.009 & 0.000 & -0.158 & -0.124 \\
\hline
\end{tabular}

Note: Robust standard errors. AZ/J\&J = AstraZeneca or Johnson \& Johnson treatment.

Again, we note that these are not causal estimates and should be taken as entirely descriptive. We cannot rule out confounding mediators or endogeneity. That being said, our data is consistent 
with the notion that safety and efficacy perceptions underlie comparative hesitancy towards the AstraZeneca and Johnson \& Johnson vaccines.

\section{$\underline{\text { References }}$}

Bullock, J. G., Green, D. P., \& Ha, S. E. (2010). Yes, but what's the mechanism? (Don't expect an easy answer). Journal of Personality and Social Psychology, 98(4), 550-558.

\section{Willingness to Wait Extension}

Our analysis shows us that the public does indeed differentiate by vaccine brand. But how intense are these preferences? One way to evaluate this is by asking respondents to make a trade off: get AstraZeneca right away and thus protection from COVID-19, or wait a period of time unprotected for their preferred vaccine. Hesitancy towards AstraZeneca may fade when there is clearly a cost to that hesitancy.

We conducted two additional surveys. The first survey was fielded from April 22-30, 2021 on a sample of 1,441 Canadian citizens 18 years or older using the online panel provider Dynata. The details of the data collection are otherwise identical to the surveys we presented above. We asked respondents to choose between receiving the AstraZeneca or Johnson \& Johnson vaccine (randomized) or a vaccine from Pfizer or Moderna (randomized). Respondents could also report that they would not take any COVID-19 vaccine. Among respondents who chose either Pfizer or Moderna, we asked them to imagine they were given a choice between AstraZeneca/Johnson \& Johnson or their preferred vaccine after a delay of some number of months. Respondents were asked their choice for a delay of 1 month through to 12 months or more.

We construct a measure to capture the maximum tolerated delay to receive their preferred vaccine. A score of 0 represents those who are unwilling to delay vaccination for their preferred vaccine and opted for AstraZeneca or Johnson \& Johnson right away, while a score of 12 indicates a respondent was willing to wait twelve months or more for their preferred vaccine rather than receive AstraZeneca or Johnson \& Johnson. A modest number of respondents did not report a consistent preference structure. For instance, they may have reported wanting an immediate vaccination with AstraZeneca or Johnson \& Johnson rather than a one month delay for their preferred vaccine, but also report wanting a 12 month delay or more for their preferred vaccine rather than receive AstraZeneca or Johnson \& Johnson right away. 17\% of respondents in this survey gave inconsistent responses in a similar manner. We exclude these respondents from our analysis as a conservative estimate of the delay people are willing to endure for their preferred vaccine. We provide the results from the full sample in Figure S5.

The second survey was fielded from May 17-28, 2021 on a sample of 1,973 Canadian adults using identical data collection procedures. This survey includes an identical task, but instead of being asked to choose between AstraZeneca/Johnson \& Johnson and Pfizer/Moderna vaccines, respondents were asked to choose between Pfizer and Moderna. Respondents who reported 
preferring Pfizer (82\% of those with a preference) were asked to report their willingness to get Moderna immediately or Pfizer after some delay, while the reverse was true for those who reported preferring Moderna.

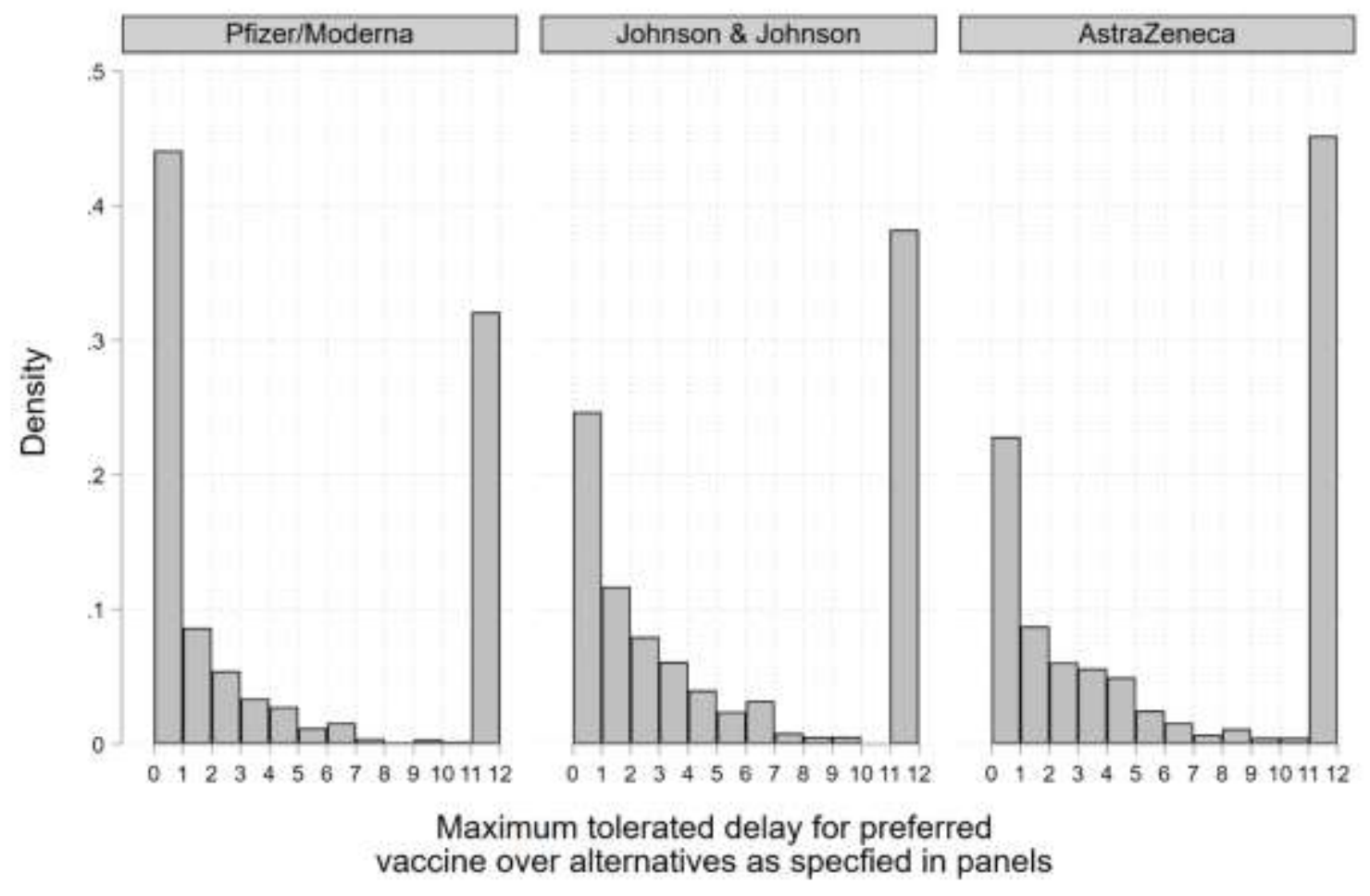

Figure S4. Histogram of maximum tolerated delay for preferred vaccine over Pfizer or Moderna (Survey 2), Johnson \& Johnson (Survey 1), and AstraZeneca (Survey 1).

Figure S4 presents a histogram of the maximum delay tolerated by respondents. It is clear that brand preferences are relatively intense, and this is particularly true when the choice is between Pfizer and Moderna on the one hand, or AstraZeneca and Johnson \& Johnson, on the other. Only $23 \%$ of respondents were willing to take AstraZeneca or Johnson \& Johnson immediately, 41\% were willing to wait 12 months or more for their preferred vaccine, while $36 \%$ were willing to tolerate some intermediate delay. These differences are not as stark when respondents were asked to choose between Pfizer and Moderna. 43\% were willing to take their less preferred vaccine immediately, while $32 \%$ reported a willingness to wait 12 months or more and $24 \%$ were willing to tolerate some intermediate delay. Importantly, even if our time experiment calibrates only coarsely to actual time preferences, we do see important differences across vaccines, suggesting that however long individuals would actually be willing to wait, it is longer when offered Astra Zeneca or Johnson and Johnson. 


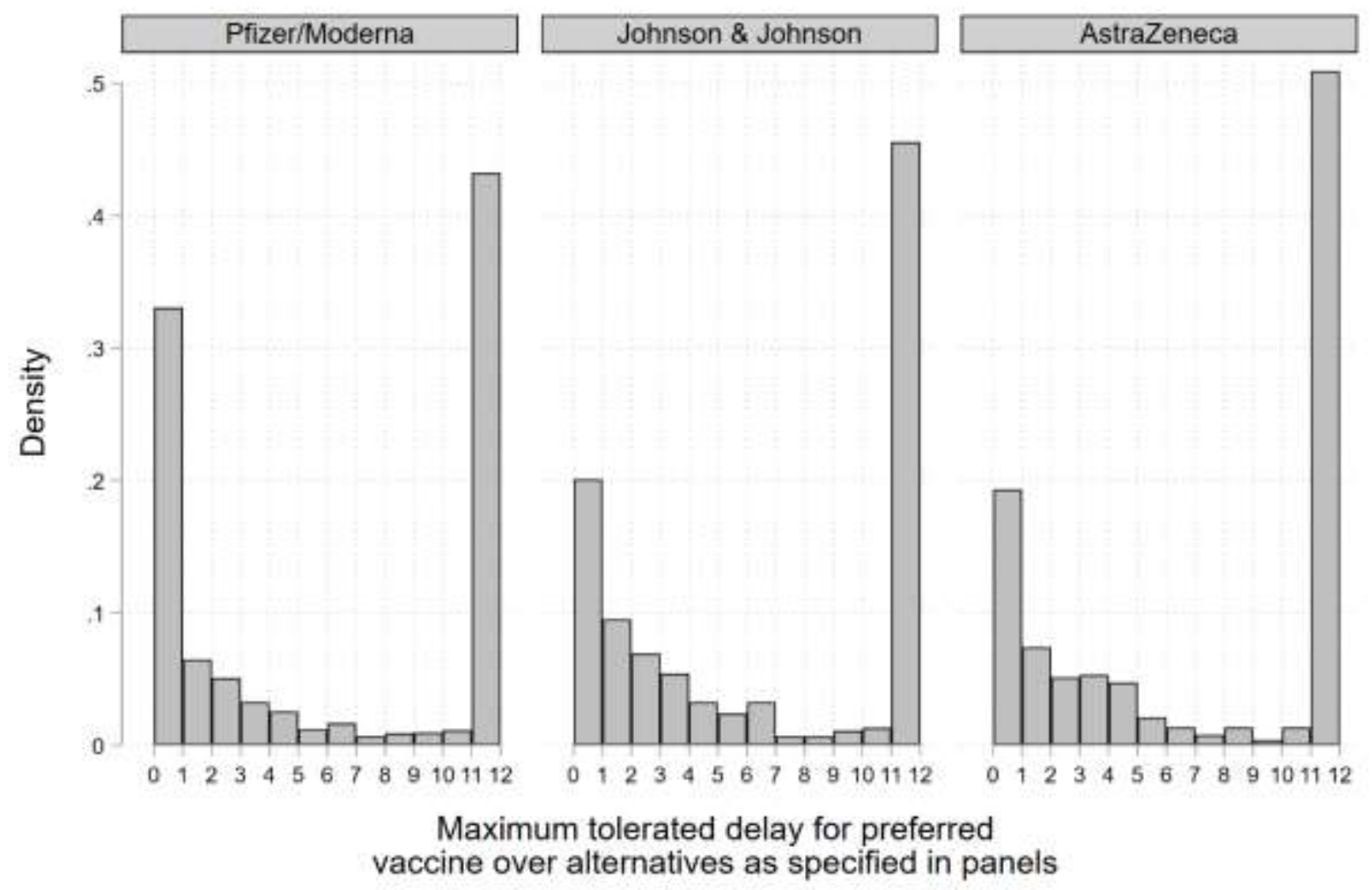

Figure S5. Histogram of maximum tolerated delay for preferred vaccine over Pfizer or Moderna (Survey 2), Johnson \& Johnson (Survey 1), and AstraZeneca (Survey 1). All respondents. 